\title{
The Short-Term and Long-Term Effects of Cardiac Resynchronization Therapy in Heart Failure Patients
}

\author{
Xuefang Zhang*, Ying Qin, Gaoxing Zhang, Weidong Gao, Yucheng Peng, Qiang Ren, Gang Sun, \\ Jinxue Liu, Bin Zhang, Juan Wu
}

Department of Cardiology, Jiangmen Central Hospital, Affiliated to Sun Yat-Sen University, Jiangmen, China

Email address:

sdfeixue81@163.com (Xuefang Zhang)

${ }^{*}$ Corresponding author

\section{To cite this article:}

Xuefang Zhang, Ying Qin, Gaoxing Zhang, Weidong Gao, Yucheng Peng, Qiang Ren, Gang Sun, Jinxue Liu, Bin Zhang, Juan Wu. The Short-Term and Long-Term Effects of Cardiac Resynchronization Therapy in Heart Failure Patients. Cardiology and Cardiovascular Research . Vol. 3, No. 1, 2019, pp. 6-9. doi: 10.11648/j.ccr.20190301.12

Received: December 14, 2018; Accepted: January 11, 2019; Published: February 19, 2019

\begin{abstract}
Objective to evaluate the short-term and long-term effects of cardiac resynchronization therapy in heart failure patients. Methods We continuous assessed forty-eight heart failure patients who underwent CRT implantation in our hospital from January 2008 to December 2012, evaluate/measure NYHA grade, left ventricular diastolic diameter (LVEDd), left ventricular ejection fraction (LVEF) and B-type natriuretic peptides (BNP) before cardiac resynchronization therapy and half year, one year, two years after cardiac resynchronization therapy. Results Compared to the results before cardiac resynchronization, half year, one year and two years later, NYHA grade, LVEF, LVEDd, BNP improved significantly $(\mathrm{P}<0.05)$; Compared to the results of half year after cardiac resynchronization therapy, one year and two years later, NYHA grade, LVEF, LVEDd, BNP also improved significantly $(\mathrm{P}<0.05)$; However, compared the results between one year and two years later after cardiac resynchronization therapy, there were no difference as to NYHA grade, LVEF, LVEDd and BNP $(\mathrm{P}>0.05)$. Conclusions CRT could improve cardiac function in patients with congestive heart failure, however, when we followed up the patients for two years, cardiac function did not improve further.
\end{abstract}

Keywords: Cardiac Resynchronization Therapy, Cardiac Function, B-Type Natriuretic Peptides

\section{Introduction}

Cardiac resynchronization therapy (CRT) was an important way for the treatment of heart failure, By "resynchronizing" cardiac contraction through restoration of inter-/intraventricular and atrioventricular dyssynchrony, CRT could reduce hospitalization for heart failure and improve survival in patients with left ventricular systolic dysfunction and wide QRS[1], These beneficial effects are related to left ventricular reverse remodeling and an improvement of left ventricular ejection fraction (LVEF) [2], Past studies had demonstrated its effectiveness in the treatment of heart failure [3], however, many studies had followed the patients for just six months, few studies have assessed left ventricular reverse remodeling over a very long period of time, Cappola TP et al[4] found CRT could promotes reverse remodeling after 6 months, Castellant $\mathrm{P}$ et al [5] followed patients for 1, 6, and 12 months after CRT implantation, found LVEF all increased, but the study only compared the LVEF of 1 month, six months, and 12 months with the LVEF before CRT implantation, there was no comparison between 1 month and six months or 1 month and 12 months after CRT implantation, So we conducted continuous follow-up for half year, one year, two years after CRT implantation, besides compared the LVEF between pre-CRT implantation and post-CRT implantation, we also compared the LVEF between each visit post-CRT implantation, aim to reveal whether cardiac function could continuously improved after CRT implantation.

\section{Methods}

\subsection{Study Population}

This was a single-center observational study. We continually enrolled 48 heart failure patients who underwent 
CRT implantation in our center from January 2008 to December 2012, all patients met the following inclusion criteria: Dilated cardiomyopathy or ischemic cardiomyopathy, New York Heart Association (NYHA) functional class III or $\mathrm{IV}$, left ventricular diastolic diameter (LVEDd) $\geq 55 \mathrm{~mm}$, left ventricular ejection fraction (LVEF) $\leq 35 \%$, in sinus rhythm, QRS $\geq 120 \mathrm{~ms}$. All patients received optimal medical therapy before CRT device implantation. The following patients were excluded: 1) patients older than 75 years, 2) patients with atrial fibrillation, 3) serum creatinine $>265 \mathrm{umol} / 1$.

\subsection{Echocardiography}

The echocardiographic analyses were performed using a GE Vivid 7 system. M-ultrasound and two-dimensional ultrasound were performed, LVEF was calculated from the conventional apical 2- and 4-chamber images, using the modified biplane Simpson's method, and the LV end-diastolic diameter was measured perpendicular to long axis of the LV at the level of the MV leaflet tips [6-7]

\subsection{B-Type Natriuretic Peptides Measurements}

Samples of EDTA-anticoagulated venous blood were obtained before CRT implantation, half year, one year, two years after CRT implantation, The BNP concentrations in plasma were determined using a 2-step microparticle enzyme immunoassay on an Abbott Ax Sym analyzer.

\subsection{CRT Implantation}

All leads were placed transvenously via the subclavian using fluoroscopy. The right ventricular lead was positioned in the apex, The right atrial lead was positioned in the appendage, Transvenous left ventricular lead positioning was guided by coronary sinus venogram, preferring a lateral or posterolateral vein as implantation site, depending on the ability to cannulate the veins, acquired acceptable pacing parameters, without diaphragmatic stimulation [8]. then we fixed the leads, connected the pulse generator (the Insync of Medtronic Company and Epic $+\mathrm{HF}$ and Frontier of St Jude Company), put the pulse generator into the subcutaneous pouch. atrial-ventricular (AV) delay and left-right ventricular (VV) delay were optimized using echocardiogram within one week after CRT implantation.

\subsection{Follow up}

NYHA class, echocardiographic variables such as LVEDd,
LVEF, the plasma concentration of BNP were recorded before CRT device implantation, and repeated at half year, one year, two years after CRT implantation.

\subsection{Statistical Analysis}

All continuous values were expressed as mean \pm standard deviation (SD). The data between groups were compared by impendent t-test, Statistical significance was assumed at a value of $\mathrm{P}<0.05$. All analysis were performed with the SPSS 13.0 software package.

\section{Results}

\subsection{CRT Implantation}

CRT were implanted in all forty-eight patients successfully, three patients experienced acute heart failure during surgery, after emergency rescue, CRT implantation was completed. In all the patients, RA and RV leads were inserted endocardially, Left ventricular leads were implanted to the posterolateral vein of coronary vein in thirty-five patients, in another thirteen patients, left ventricular leads were implanted to the lateral vein, Pacing and capturing function test were all well during surgery. There were no complication during surgery such as cardiac tamponade, diaphragmatic muscle stimulation and so on.

\subsection{Follow up}

We completed two years follow up in forty patients, four patients were followed up for one year, two patients were followed up only for half years, two patients missed.

\subsection{NYHA Class, Echocardiography Parameters, BNP}

Compared to the parameters before CRT implantation, half year, one year, two years after CRT implantation, NYHA class all improved, LVEF increased, LVEDd decreased, the plasma concentration of BNP deceased ( $\mathrm{P}$ all $<0.05$ ). Compared to the parameters after half year of CRT implantation, one year, two years after CRT implantation, NYHA class all improved, LVEF increased, LVEDd decreased, the plasma concentration of BNP deceased ( $\mathrm{P}$ all $<0.05$ ). Compared between the parameters of one year and two years after CRT implantation, there were no significant difference as to NYHA class, LVEF, LVEDd, and the plasma concentration of BNP (P all $>0.05)$. Table 1 .

Table 1. NYHA class, echocardiography parameters, BNP before CRT, half year after CRT, one year after CRT, two years after CRT.

\begin{tabular}{llll}
\hline & pre-CRT & half year after CRT & one year after CRT \\
\hline NYHA & $3.6 \pm 0.2$ & $3.0 \pm 0.2^{\mathrm{a}}$ & $2.8 \pm 0.3^{\mathrm{ab}}$ \\
LVED $(\mathrm{mm})$ & $72.4 \pm 8.3$ & $70.3 \pm 9.6^{\mathrm{a}}$ & $66.0 \pm 11.7^{\mathrm{ab}}$ \\
LVEF $(\%)$ & $28.6 \pm 9.7$ & $33.4 \pm 11.8^{\mathrm{a}}$ & $38.4 \pm 10.9^{\mathrm{ab}}$ \\
BNP $(\mathrm{pg} / \mathrm{ml})$ & $12630.3 \pm 3129.8$ & $6570.7 \pm 2320.5^{\mathrm{a}}$ & $3877.2 \pm 1110.5^{\mathrm{ab}}$ \\
\hline
\end{tabular}

${ }^{\text {a }} P<0.05$ compared to pre-CRT; ${ }^{\text {b }} P<0.05$ compared to half year after CRT

\section{Discussion}

The findings in the current study can be summarized as follows: 1) CRT could improve cardiac function after six months, one year, two years; 2) Compared to six months after after CRT implantation, cardiac function could improve 
continuously in one year and two years; 3) However, cardiac function did not continuously improve between one year and two years, as NHHA class, LVEF, LVEDd, BNP did not improved in two years after CRT implantation when compared to one year after CRT implantation.

Nowadays, congestive heart failure remained difficult to handle with medical treatment, morbidity and mortality remained high, with the application of angiotensin converting enzyme inhibitors, angiotensin receptor antagonist, $\beta$-blockers, aldosterone receptor antagonist in clinical practice, the prognosis of heart failure had improved, however, there still some patients remained bad prognosis [9-10]. Often, There were dsynchronization between atrial-ventricular, inter ventricular, intra-ventricular in heart failure patients, while cardiac resynchronization therapy, besides the traditional pacing of right atrial and right ventricular, it adds left ventricular pacing, 'so could restore the synchronization of the heart, increase cardiac output. Past studies had demonstrated that CRT could improve cardiac function, decreased mortality in heart failure patients [11]. St John Sutton M G et al [12] studied 323 patients, 172 patients were randomized to CRT group and 151 patients randomized to the control group, after 6 months, CRT was associated with reduced end-diastolic and end-systolic volumes, increased ejection fraction, this study just followed up the patients for 6 months. Gaspirini et al [13] have demonstrated in a population of 48 patients with CRT that the greatest changes in LVEF and reverse remodeling usually occurred within the first 6 months but can continue $\geq$ 2 years. In that study, LV end-systolic diameter decreased by approximately $35 \%$ at 12 months, but continued LV reverse remodeling occurred after 12 months allowing the LV end-systolic diameter to decrease by $\geq 50 \%$ of the initial volume. However, they did not compared LVEF and LV end-systolic diameter between 6 months, one year and two years. Our study conducted continuous follow-up in heart failure patients with CRT implantation, We found LVEDd decreased significantly after six months, one year and two years of CRT implantation, LVEF increased significantly. Compared to half year after CRT implantation, one year and two years later after CRT implantation, LVEDd decreased and LVEF increased further, while compared to one year after CRT implantation, left ventricular diameter, LVEF did not improved further in two years after CRT implantation, implies that CRT could not improve cardiac function continuously.

Past studies found that, CRT could improve neurohormonal environment, reversed cardiac remodeling in the long time, which were independent of each cardiac cycle, even suddenly turn off CRT, the improvement of heart function will last for a certain time. At the same time, CRT was the backup support for drugs titrated to the maximum dose, especially for beta blockers. researches have found that about $50 \%$ heart failure patients who can not tolerate drugs therapy could tolerate drugs therapy after CRT implantation, the dose could be titrated [14]. Muraoka $H$ et al [15] reported a HF patient with left bundle branch block due to nonischemic cardiomyopathy who received CRT implantation, $20 \mathrm{mg}$ /day carvedilol was administered, after one year, LVEF improved partially from 7\% to $18 \%$. HF symptom remained unchanged (NYHA class III), then the dose of carvedilol was titrated up to $40 \mathrm{mg} / \mathrm{day}$, LVEF increased up to $34 \%$ along with improvement in the HF symptom (NYHA class I). Our studies found CRT along with drugs therapy could improve cardiac function, It's worth noting that with the time extension after CRT implantation, cardiac function did not improved further, the possible reason maybe that drugs doses had reached to a target dose.

BNP and NT-proBNP levels are increased in heart failure, and correlate well with ventricular wall stress and severity of heart failure. Elevation of these peptides has a well established role for prediction of mortality for both acute and chronic heart failure situations [16-17]. Bakos Z et al [18] found that BNP levels were significantly reduced in CRT responders compared to non-responders, and that the greatest reduction was seen in those patients who exhibited both a clinical and echocardiographic positive response. In contrast, patients with no BNP reduction had a poor prognosis with significantly higher mortality and heart failure hospitalizations.

Our study found, BNP decreased significantly after half year, one year and two years after CRT implantation, implies cardiac function improved. While BNP did not decreased compared between one year and two years after CRT implantation, implies between one year and two years after CRT implantation, cardiac function did not improved, this was in accordance with echocardiography.

Study limitations

The first limitation of the present study is that this is a single center study and the number of patients included is relatively small. Secondly, Clinical response was based only in NYHA class improvement and did not include functional capacity evaluation and quality of life scores, and the definition of LV remolding used was limited to change in LV end diastolic dimensions, while LV volumes were not available. Thirdly, we did not conducted atrioventricular and interventricular optimization between one year and two years after CRT implantation, this may result in no improvement of cardiac function in two years after CRT.

\section{Conclusion}

CRT could improve the short term cardiac function in patients with hear failure, as half year after CRT implantation, cardiac function improved, and the improvement continued in the following one year and two years, as NYHA, LVEF, LVEDd, BNP all improved when compared to half year after CRT implantation, however, cardiac function did not improved continuously between one year and two years, as NYHA, LVEF, LVEDd, BNP did not improved compared to one year after CRT implantation. The sample size of our study was few, there were more challenges as for CRT application in clinical practice, large sample studies were needed in the future to demonstrated the efficacy of CRT in heart failure patients. 


\section{Conflict of Interest}

The authors declare no conflicts of interest.

\section{References}

[1] Moss AJ, Hall WJ, Cannom DS, et al. Cardiac-Resynchronization Therapy for the Prevention of Heart-Failure Events. $N$ Engl $J$ Med, 2009, 361 (14):1329-1338.

[2] Ruwald MH, Solomon SD, Foster E, et al. Left Ventricular Ejection Fraction Normalization in Cardiac Resynchronization Therapy and Risk of Ventricular Arrhythmias and Clinical Outcomes: Results from the MADIT-CRT Trial. Circulation, 2014, 2278-2287.

[3] Bristow MR, Saxon LA, Boehmer J, et al. Cardiac-Resynchronization Therapy with or without an Implantable Defi brillator in Advanced Chronic Heart Failure. N Engl J Med, 2004, 350 (21):2140-2150.

[4] Cappola TP, Harsh MR, Jessup M, et al. Predictors of remodeling in the CRT era: influence of mitral regurgitation, BNP, and gender. J Card Fail, 2006, 12:182-188.

[5] Castellant P, Fatemi M, Orhan E, Etienne Y, Blanc JJ. Patients with non-ischaemic dilated cardiomyopathy and hyper-responders to cardiac resynchronization therapy: characteristics and long-term evolution. Europace, 2009, $11: 350-5$.

[6] Lang RM, Badano LP, Mor-Avi V, Afilalo J, Armstrong A, Ernande L, Flachskampf FA, et al. Recommendations for cardiac chamber quantification by echocardiography in adults: an update from the American Society of Echocardiography and the European Association of Cardiovascular Imaging. J Am Soc Echocardiogr, 2015, 28:1-39.

[7] Shiller NB, Shah PM, Crawford M, et al. American Society of Echocardiography committee on standards, Subcommittee on quantitation of Two-Dimensional Echocardiograms: Recommendations for quantitation of the left ventricle by two-dimensional echocardiography. J Am Soc Echocardiogr, 1989, 2:358-367.

[8] Dong YX, Powell BD, Asirvatham SJ, Friedman PA, Rea RF, Webster TL, Brooke KL, Hodge DO, Wiste HJ, Yang YZ, Hayes DL, Cha YM. Left ventricular lead position for cardiac resynchronization: a comprehensive cinegraphic, echocardiographic, clinical, and survival analysis. Europace, 2012, 14:1139-1147.
[9] Khand A, Gemmel I, Clark AL, et al. Is the prognosis of heart failure improving?. J Am Coll Cardiol, 2000, 36 (7):2284-2286.

[10] Cleland JGF, Clark AL. Delivering the cumulative benefi ts of triple therapy to improve outcomes in heart failure: too many cooks will spoil the broth. J Am Coll Cardiol, 2003, 42 (7):1234-1237.

[11] Cleland JG, Daubert JC, Erdmann E, et al. The effect of cardiac resynchronization on morbidity and mortality in heart failure. N Engl J Med, 2005, 352 (12):1539-1549.

[12] St John Sutton MG, Plappert T, Abraham WT, et al. Effect of cardiac resynchronization therapy on left ventricular size and function in chronic heart failure. Circulation, 2003, 107:1985-1990.

[13] Gasparini M, Auricchio A, Regoli F, et al. Four-year efficacy of cardiac resynchronization therapy on exercise tolerance and disease progression: the importance of performing atrioventricular junction ablation in patients with atrial fibrillation. J Am Coll Cardiol, 2006, 48:734-43.

[14] Aranda JM Jr, Woo GW, Conti JB, et al. Use of cardiac resynchronization therapy to optimize betablocker therapy in patients with heart failure and prolonged QRS duration. Am J Cardiol, 2005, 95 (7):889-891.

[15] Muraoka H, Imamura T, Kinugawa K. Reverse remodeling achieved by combination therapy with high-dose beta blocker and cardiac resynchronization. Int Heart J, 2015, 56: 462-465.

[16] Oremus M, Don-Wauchope A, McKelvie R, Santaguida PL, Hill S, Balion C, et al. BNP and NT-proBNP as prognostic markers in persons with chronic stable heart failure. Heart Fail Rev, 2014, 19 (4):471-505.

[17] Santaguida PL, Don-Wauchope AC, Oremus M, McKelvie R, Ali U, Hill SA, et al. BNP and NT-proBNP as prognostic markers in persons with acute decompensated heart failure: a systematic review. Heart Fail Rev, 2014, 19 (4):453-70.

[18] Bakos Z, Chatterjee NC, Reitan C, Singh J P, Borgquist R. Prediction of clinical outcome in patients treated with cardiac resynchronization therapy - the role of NT-ProBNP and a combined response score. BMC Cardiovascular Disorders, 2018, 18:70. 